\title{
Ligadura del cordón umbilical en recién nacidos prematuros
}

\author{
Umbilical cord clamping in preterm infants
}

\author{
Dr. José M. Ceriani Cernadas ${ }^{a, b, c}$
}

\begin{abstract}
RESUMEN
La prematuridad fue aumentando la supervivencia desde hace varios años, y eso produce, sobre todo, una preocupación en los prematuros nacidos antes de las 28 semanas de gestación. El tiempo del clampeo del cordón umbilical puede generar diversos trastornos, principalmente, cuando se realiza temprano (10-15 segundos). Ya desde hace 20 años, a través de varias investigaciones, se pudieron demostrar los notorios beneficios del clampeo demorado del cordón (de 2 a 3 minutos). Esta práctica fue instalada en la asistencia obstétrica y neonatal por las recomendaciones de sociedades científicas y de las revisiones sistemáticas, que señalaron las sólidas evidencias que apoyaban esta conducta para prematuros. En esta revisión, se describen los artículos más relevantes en los últimos años, que sustentan notoriamente la aplicación del clampeo demorado del cordón versus el clampeo temprano. Asimismo, esta práctica genera una disminución de los trastornos graves en prematuros.

Palabras clave: clampeo, cordón umbilical, circulación placentaria, recién nacido prematuro.
\end{abstract}

http:/ / dx.doi.org/10.5546/ aap.2021.e315

Texto completo en inglés:

http:/ / dx.doi.org/10.5546/ aap.2021.eng.e315

a. Consejo de Publicaciones y Biblioteca, Sociedad Argentina Pediatría.

b. Comité de Seguridad del Paciente, Hospital Italiano de Buenos Aires.

c. Honorario en Servicio de Neonatología, Hospital Italiano de Buenos Aires.

Correspondencia: Dr. José M. Ceriani Cernadas:

jose.ceriani@

hospitalitaliano.org.ar

Financiamiento:

Ninguno.

Conflicto de intereses:

Ninguno que declarar.

Recibido: 7-9-2020

Aceptado: 10-12-2020
Cómo citar: Ceriani Cernadas JM. Ligadura del cordón umbilical en recién nacidos prematuros. Arch Argent Pediatr 2021;119(4):e315-e321.

El tiempo de la ligadura del cordón umbilical se ha tenido en cuenta desde hace siglos. Hasta terminada la Segunda Guerra Mundial, los nacimientos solían ocurrir en casas familiares, y el cordón se cortaba cuando dejaba de latir. Algunas familias no realizaban la ligadura del cordón, y salía junto con la placenta. El mantener el latido del cordón lo efectuó el ser humano a través de miles de siglos, algo confirmado en los Homo sapiens. También ocurre en mamíferos, animales que se parecen mucho a nosotros.
En los primeros años de este siglo, el clampeo demorado del cordón fue aumentando su frecuencia. Eso se debió a estudios científicos realizados en recién nacidos. ${ }^{1-11}$ La mayoría de los países comenzaron a efectuar esa acción y observaron que los neonatos con clampeo demorado tenían una mejor evolución a largo plazo. ${ }^{2}$

$\mathrm{Al}$ principio, se publicaron algunos estudios relacionados con el tiempo del cordón en prematuros, en escasos neonatos. En un estudio piloto, reclutaron a 32 prematuros nacidos entre las 24 y las 32 semanas, cuyas ligaduras se habían producido entre 5 y 10 segundos vs. 30 y 45 segundos. Los resultados revelaron que el grupo con clampeo demorado tenía una presión sanguínea más elevada y no se necesitaba administrar oxígeno. ${ }^{12}$

Otros estudios incluyeron a prematuros nacidos antes de las 37 semanas de gestación, con una población mayoritaria de entre 34 y 36 semanas, y el resultado del clampeo del cordón era similar al de los nacidos a término.

La evolución del clampeo del cordón mejoró a través de la revisión sistemática Cochrane en el año 2007, que tomó siete estudios con 297 prematuros, del total de 16 identificados. El principal objetivo evaluó el tiempo de transfusión placentaria, acorde al momento del clampeo del cordón. Las variables de resultados fueron transfusión, anemia y hemorragia intraventricular, cuya incidencia resultó significativamente menor en los neonatos con clampeo entre 30 y 120 segundos. ${ }^{13}$

Luego de la revisión sistemática, se publicaron otros estudios clínicos relacionados con clampeo demorado, que evaluaron la transfusión 
placentaria. En uno, observaron que el aumento de glóbulos rojos evitaba la anemia, un trastorno muy frecuente. Esta se fue produciendo en las frecuentes extracciones de sangre en las unidades de cuidados intensivos neonatales (UCIN). La mayoría de los prematuros menores de 32 semanas, habitualmente, reciben varias transfusiones de sangre. Asimismo, la anemia interfiere en la evolución de enfermedades respiratorias (en especial, displasia broncopulmonar) y la infección bacteriana por transfusiones de sangre. ${ }^{4-16}$

En otra revisión Cochrane en junio de 2012, se agregaron 15 estudios. ${ }^{17}$ Incluyeron un estudio que definió el mejor resultado generado por clampeo demorado versus el clampeo inmediato. Con el clampeo demorado, los prematuros tuvieron menos transfusiones de sangre por anemia (7 ensayos, 392 prematuros). Además, presentaron una menor incidencia de hemorragia intraventricular (10 ensayos, 539 neonatos) y enterocolitis necrotizante (5 ensayos, 241 neonatos). ${ }^{18,19}$

Otro estudio evaluó la relación del clampeo inmediato y el demorado en prematuros nacidos $\mathrm{a} \leq 32$ semanas gestacionales. El resultado mostró que el clampeo demorado produjo una reducción significativa de hemorragia intraventricular y transfusiones tempranas. ${ }^{20}$

Diversos estudios evaluaron resultados de la ligadura demorada del cordón y observaron los beneficios neonatales, tales como mejor circulación de transición, mejor establecimiento del volumen de glóbulos rojos, menor necesidad de transfusión de sangre, menor incidencia de enterocolitis necrotizante y menos hemorragia intraventricular. Los niveles de bilirrubina fueron más altos en los prematuros con clampeo demorado, sin diferencias estadísticamente significativas en la fototerapia entre los grupos. En otros resultados, muerte neonatal, hemorragia intraventricular grave (grado 3-4) y leucomalacia periventricular no tuvieron diferencias entre los grupos. Concluyeron que la transfusión placentaria con clampeo demorado mejoraba la presión arterial, reducía la transfusión de sangre, disminuía el riesgo de hemorragia intraventricular y de enterocolitis necrotizante. ${ }^{21-23}$

Ambas revisiones Cochrane, la de 2007 y la de 2012, contribuyeron enormemente a aumentar el conocimiento sobre los efectos del tiempo de ligadura del cordón en los prematuros. Los resultados pudieron señalar los beneficios del clampeo demorado, especialmente, en la transfusión placentaria fisiológica. En ninguno de los estudios evaluados en revisiones publicadas, se observaron eventos adversos atribuibles al clampeo demorado. Este último aspecto tiene una enorme trascendencia en la medicina, ya que siempre debe comprobarse que toda práctica sea segura antes de aplicarla y solo así podrá incorporarse.

El mayor desafío en los últimos años fue conocer qué sucedía con el tiempo del clampeo del cordón en los prematuros extremos. Observaron que el pasaje de sangre luego del primer minuto contribuía a una mejor reanimación en los prematuros que tuvieron un muy bajo Apgar al nacer. Estos aspectos son de importancia, ya que los prematuros extremos tienen una mayor morbilidad y mortalidad en el primer año de vida $y$, con frecuencia, presentan secuelas en el neurodesarrollo, que persisten a largo plazo y pueden perdurar toda la vida.

El Colegio Estadounidense de Obstetras y Ginecólogos (American College of Obstetricians and Gynecologists) realizó recomendaciones respecto al momento del clampeo del cordón. Señaló que el clampeo tardío del cordón se asociaba con beneficios significativos en los prematuros, mejor circulación de transición, mejor establecimiento del volumen de glóbulos rojos, menor necesidad de transfusión de sangre, menor incidencia de enterocolitis necrotizante y de hemorragia intraventricular. Recomendó que el tiempo del clampeo podría permanecer entre 2 y 5 minutos después del nacimiento, y señaló que el pasaje de sangre desde el primer minuto podía contribuir a una mejor reanimación en los prematuros con muy bajo Apgar. ${ }^{24}$

Diversos aspectos están presentes en los prematuros, especialmente, la hipotermia, que puede generar graves consecuencias y, por lo tanto, se debe evitar en los prematuros extremos. Esta puede ocurrir cuando se espera el tiempo de ligadura demorada y en la reanimación. Para que eso no suceda, es imprescindible que, al nacer, se envuelva al prematuro rápidamente en una bolsa de plástico sin secarlo y se le deje un gorro de tela en la cabeza. Estas medidas son efectivas para mantener la temperatura en los rangos normales y un mejor acceso a la vía aérea.

La respiración es débil en los prematuros extremos, y es necesario aplicar presión positiva mediante bolsa y máscara para una recuperación adecuada. En ciertos momentos, se puede realizar la intubación endotraqueal en la sala de partos, aunque es poco frecuente. Asimismo, 
el clampeo demorado aumenta la transfusión placentaria y contribuye a que el flujo umbilical venoso y arterial genere una mayor expansión de los pulmones, mediante el aumento del lecho vascular pulmonar, y esto facilita la recuperación del neonato. ${ }^{25,26}$

Otro estudio evaluó el efecto del clampeo tardío en la respiración, mediante la transición al nacer con $\leq 29$ semanas. Hubo 62 neonatos en el grupo del clampeo demorado y 62 en el de clampeo inmediato. Al compararlos, entre los que tuvieron clampeo temprano, algunos no respiraban espontáneamente y debieron ser intubados $(\mathrm{p}=0,01)$; hubo una mayor frecuencia de enfermedad pulmonar crónica $(\mathrm{p}=0,02)$ y hemorragia intraventricular grave $(p=0,02) .{ }^{27}$

En una investigación realizada en Australia, observaron que entre el clampeo temprano y el demorado no había diferencias significativas, excepto en la mortalidad, que fue significativamente más elevada en los neonatos con clampeo temprano. ${ }^{28}$ En otro estudio evaluaron las consecuencias clínicas al efectuar el clampeo demorado en prematuros moderados y tardíos. Los resultados señalaron que el hematocrito al nacer resultó mayor en forma significativa en el clampeo demorado versus el clampeo temprano. Asimismo, hubo menos prematuros con clampeo demorado en la UCIN, en comparación con el clampeo temprano $(p=0,04)$. La incidencia del síndrome de dificultad respiratoria fue significativamente menor en el grupo demorado versus el clampeo temprano $(\mathrm{p}=0,002){ }^{29}$

En otro estudio, observaron a prematuros con clampeo demorado vs. clampeo inmediato. Los resultados mostraron menos hemorragia intraventricular y adecuada temperatura axilar, que fue significativamente superior en los que recibieron clampeo demorado. No encontraron diferencias en las puntuaciones de Apgar a 1 y 5 minutos, el nivel de bilirrubina a las 24 horas y el del hematocrito. ${ }^{30}$

Otra investigación encontró que, con el clampeo demorado, podían detectar mejor la aloinmunización de glóbulos rojos, que generaba la enfermedad hemolítica inmune del recién nacido, que producía anemia e ictericia neonatal. Se compararon dos grupos, uno con clampeo temprano durante el primer período del estudio (enero de 2001-junio de 2009) y otro con clampeo demorado (junio de 2009-diciembre de 2014). El resultado primario fue la necesidad de transfusión de sangre después del nacimiento. La hemoglobina al nacer fue significativamente más alta en el grupo demorado $(p=0,0003)$, que no tenía anemia al nacer $(p=0,004)$. Además, el clampeo tardío del cordón demostró una notoria mejoría en los niveles de hemoglobina, y así disminuyeron los riesgos de la aloinmunización en glóbulos rojos, con una mejor tasa de hemoglobina y menor necesidad de transfusiones. ${ }^{31}$

Una apropiada revisión sistemática y un metaanálisis detectaron 18 ensayos controlados, aleatorizados, y compararon el clampeo tardío del cordón vs. el clampeo temprano, en 2834 prematuros. El clampeo tardío redujo la mortalidad en forma significativa, que fue similar en los prematuros nacidos con $\leq 28$ semanas de gestación. ${ }^{32}$

En un estudio, evaluaron diferencias del clampeo en los primeros segundos vs. el clampeo demorado. Se realizó en ocho unidades de maternidades terciarias del Reino Unido. Participaron 276 prematuros nacidos antes de las 32 semanas. Observaron que había una evidencia superior al mantener el clampeo a los 2 minutos, con mejores resultados. ${ }^{33}$

En otro estudio, detectaron datos sobre los efectos de intervenciones del cordón umbilical en madres que tuvieron cesáreas. No hubo diferencias significativas en la mortalidad, pero la hemorragia intraventricular grave fue significativamente mayor en el grupo con ligadura temprana del cordón. La prueba de interacción entre los estratos de edad gestacional y el grupo de tratamiento fue significativa para la hemorragia intraventricular grave $(p=0,003) .{ }^{34}$

En los recién nacidos asfixiados, es imprescindible que la transfusión placentaria lleve a una recuperación rápida, y esto pudo observarse en un estudio con neonatos prematuros y a término. Aquellos con clampeo temprano tuvieron trastornos cardíacos y saturación de oxígeno muy baja, por lo cual requirieron más oxígeno en los primeros cinco minutos de vida. En comparación con ese resultado, el clampeo demorado demostró mejoras en la perfusión sistémica y cerebral, lo que sugirió beneficios en los neuroprotectores. También mejoraron la frecuencia cardíaca, la presión arterial, la oxigenación cerebral, el aumento en la hemoglobina y la predicción de anemia. ${ }^{35}$

Otro trastorno en los prematuros suele estar presente debido al escaso depósito de hierro, y eso ocurre más en aquellos con clampeo inmediato del cordón. No obstante, 
se observó un incremento en el hierro asociado al clampeo demorado, y se pudo conocer que esos depósitos iban aumentando a través de una mayor transfusión placentaria. Diversos estudios publicados en estos últimos años pudieron ver los conocimientos de los depósitos del hierro en los prematuros.

En uno de ellos, estimaron el efecto de la transfusión placentaria en dos grupos, con clampeo temprano (10 segundos) vs. clampeo demorado (60 segundos) en los prematuros de 30 a 33 semanas. Sus resultados mostraron que la mediana de la ferritina sérica fue significativamente mayor en el grupo de transfusión placentaria con clampeo demorado. ${ }^{36}$ Otros detectaron la incidencia de anemia a los tres meses, y el resultado mostró que, en forma significativa, fue menor en el grupo de transfusión placentaria tardía. ${ }^{37}$ En otro estudio, efectuado en nacidos por cesárea electiva, observaron que aquellos con clampeo demorado tuvieron mayores reservas de hierro a los 4 meses de edad, en comparación con el clampeo temprano. ${ }^{38}$

En otra investigación, evaluaron los efectos de ligaduras del cordón en reservas del hierro en bebés que tenían 3 meses de edad, nacidos de madres anémicas. Los neonatos de madres con hemoglobina $<100 \mathrm{~g} / 1$ fueron asignados al azar al momento del parto, ya sea por clampeo inmediato del cordón o clampeo demorado. Las medidas del resultado señalaron que las tasas de hemoglobina y la ferritina sérica a los 3 meses fueron significativamente más altas en el grupo con clampeo demorado. También observaron que las probabilidades de anemia $(<100 \mathrm{~g} / \mathrm{l})$ a los 3 meses fueron 7,7 veces más altas en el grupo de clampeo temprano, en comparación con el tardío. ${ }^{39}$

En otro estudio, el objetivo fue investigar, en una población de alto riesgo, los efectos del clampeo tardío del cordón vs. el temprano, los niveles de hemoglobina y ferritina a los 8 y a los 12 meses de edad. Los resultados en 540 lactantes mostraron que, con el clampeo tardío del cordón, había niveles más altos de hemoglobina, y así se redujo la anemia a los 8 y a los 12 meses de edad. Esto generó efectos muy positivos en la salud y en el desarrollo de los bebés. ${ }^{40}$ De estos interesantes estudios, se puede concluir que los prematuros extremos pueden compensar los escasos depósitos de hierro que fisiológicamente tienen.

Diversas investigaciones publicadas en estos años calcularon la relación de exprimir el cordón umbilical (cord milking) con las habituales ligaduras tempranas y tardías. En un estudio, los resultados señalaron que el apriete del cordón umbilical tenía ventajas con el clampeo demorado. ${ }^{41}$ No obstante, en otro estudio con mayor número de prematuros, encontraron que no hubo ninguna diferencia estadísticamente significativa en los resultados entre los grupos que exprimieron el cordón y aquellos con clampeo demorado, tanto en transfusiones placentarias como en enterocolitis necrotizante y hemorragia intraventricular. ${ }^{42}$

En un ensayo clínico aleatorizado de no inferioridad, determinaron si las tasas de muerte o hemorragia intraventricular grave diferían entre los recién nacidos prematuros que recibían transfusión placentaria a través de comprimir el cordón umbilical y los que tenían clampeo tardío. En nueve centros médicos universitarios de cuatro países, reclutaron, entre junio de 2017 y septiembre de 2018, a prematuros nacidos entre las 23 y las 31 semanas de gestación. El resultado primario fue un compuesto de muerte o hemorragia intraventricular grave para determinar la no inferioridad de apretar el cordón umbilical. No hubo diferencias en la mortalidad, pero la hemorragia intraventricular grave fue significativamente más frecuente al exprimir el cordón, en relación con el clampeo demorado. ${ }^{43}$

En el año 2020, se publicó una revisión sistemática y un metaanálisis que abordaron aspectos de sumo interés al aumentar la transfusión placentaria como un pasaje de sangre desde la placenta al recién nacido. Se incluyeron 19 estudios (2014 prematuros). Cinco $(n=922)$ compararon el cordón umbilical apretado con el clampeo demorado, y $14(\mathrm{n}=1092)$ compararon el apretar el cordón con el clampeo inmediato. El apriete del cordón aumentó significativamente el riesgo de hemorragia intraventricular en relación con el clampeo demorado. En la comparación del clampeo inmediato con el apriete del cordón, disminuyó la necesidad de transfusiones de glóbulos rojos, pero no mejoraron los resultados clínicos. Concluyeron que apretar el cordón no podía considerarse una estrategia de transfusión placentaria en los neonatos prematuros, mediante la evidencia actual. ${ }^{44}$

\section{Aspectos fisiológicos relacionados con el clampeo del cordón umbilical en los prematuros}

Los beneficios con el clampeo demorado son producidos por hechos fisiológicos, por lo cual no se considera como un tratamiento, debido a que la práctica respeta los aspectos naturales. 
Esos beneficios resultan en que el neonato reciba el volumen de sangre que le corresponde. La transfusión placentaria es una inevitable consecuencia fisiológica en los primeros minutos de vida, en la redistribución de sangre entre la placenta y el neonato. ${ }^{45,46}$ La naturaleza determinó que ese volumen de sangre le pertenecía al recién nacido; entonces, ¿por qué privarlo de que lo reciba?

Es necesario conocer que, cuando ocurre un sufrimiento fetal en etapas avanzadas del parto, el período expulsivo produce un menor pasaje de sangre hacia el feto, por lo cual es imprescindible que, al nacer, se efectúe el clampeo demorado del cordón y que el neonato reciba la sangre de la placenta y pueda recuperarse. Como ya se señaló, durante muchos años, el clampeo inmediato del cordón umbilical fue el estándar. En esa acción, totalmente inadecuada y arbitraria, no tuvieron en cuenta que el fundamento fisiológico relacionado con el clampeo del cordón era solo esperar el suficiente tiempo en la transición circulatoria desde la placenta al recién nacido. Asimismo, mejoraba la aireación de los pulmones y generaba un aumento en el flujo sanguíneo pulmonar de regreso al centro de la transición circulatoria.

Ciertas revisiones pudieron señalar los significativos beneficios de las acciones hemodinámicas con el clampeo demorado, ya que se consideraba que era un efecto protector fisiológico. También diversos estudios observaron que el tiempo en que se ligaba el cordón tenía una relación con el momento en que se producía la primera respiración, junto con otros mecanismos.

En la Argentina, a través de una iniciativa de UNICEF, se realizó, en 1999, un estudio en 31 maternidades de todo el país con el objetivo de conocer cuál era el momento en que se ligaba el cordón umbilical. Se midió el momento exacto del clampeo con cronómetro en 3738 nacimientos. La mediana del tiempo de la ligadura fue de 35 segundos en la población global. A partir de esos datos, se iniciaron una serie de acciones para promover el clampeo demorado en nuestro país.
Otro tema de importancia asociado a la fisiología es el de las células madre (stem cells), que tienen múltiples funciones y actúan sobre los aspectos madurativos, en acción antiinflamatoria en el sistema nervioso central y en la reducción de trastornos o enfermedades ${ }^{47}$ (Tabla 1 ). Asimismo, dado que se conocía que la sangre del cordón umbilical contenía varias células madre hematopoyéticas precursoras de células endoteliales en el mesénquima y células madre de linaje muy potente, se concluyó el mérito del clampeo demorado del cordón. Un importante beneficio de esa demora se relaciona con mayor cantidad de células madre en la sangre del cordón umbilical, especialmente, en los prematuros nacidos antes de las 28 semanas.

Se puede enfatizar que, en la acción de la demora en el clampeo del cordón, se observa la importancia de la transfusión placentaria al contener la sangre junto con las células madre que ingresan al neonato. Eso permite un pasaje sumamente mayor de células madre, en especial, cuando la demora es desde 60 a 120 segundos luego del nacimiento (Tabla 2).

Hace poco tiempo, se observó la primera transferencia de células madre de la humanidad y el trasplante de células madre de la naturaleza, que se publicó en el año 2010. ${ }^{48}$ Eso pudo realizarse porque, al nacer, la placenta y el cordón umbilical comenzaban a contraerse y a bombear sangre hacia el neonato.

Tabla 1. Acción de las células madre ante diversos trastornos neonatales ${ }^{48}$

\footnotetext{
Beneficios en trastornos confirmados

- Síndrome de dificultad respiratoria.

- Anemia de la prematuridad.

- Hemorragia intraventricular.

- Sepsis.

- Leucomalacia periventricular.

Beneficios en trastornos aún no confirmados

- Enfermedad pulmonar crónica.

- Apneas de la prematuridad.

- Retinopatía del prematuro.

- Enterocolitis necrotizante.

- Ductus arteriosus persistente.
}

TABla 2. Tiempo de ligadura del cordón umbilical y células madre ${ }^{47}$

\begin{tabular}{lccc}
\hline & $\begin{array}{c}\text { Tiempo de clampeo } \\
\text { (segundos) }\end{array}$ & $\begin{array}{c}\text { Volumen sanguíneo } \\
(\mathbf{m l})\end{array}$ & $\begin{array}{c}\text { Células madre hematopoyéticas } \\
\text { que recibe el neonato }\end{array}$ \\
\hline Clampeo temprano & 5 & 0 & 0 \\
Clampeo demorado & 180 & $75-80$ & $1100-45000$ \\
\hline
\end{tabular}


Aquellos que aún efectúan el clampeo temprano no conocen la fisiología de la transición en el nacimiento, lo que significa que restringen las posibilidades del trasplante de células madre y los privan de las células adicionales de la madre. Las acciones de las células madre son sumamente importantes y actúan en múltiples situaciones de riesgo, y sus efectos beneficiosos se prolongan a las edades mayores e incluso a los adultos. Asimismo, los fundamentos de sus mecanismos fisiológicos van tomando cada vez más atención al generar un aumento de las células.

\section{REFERENCIAS}

1. Van Rheenen P, Brabin BJ. Late umbilical cord-clamping as an intervention for reducing iron deficiency anaemia in term infants in developing and industrialised countries: a systematic review. Ann Trop Paediatr. 2004;24 (1):3-16.

2. Philip AG, SaigalS. When Should We Clamp the Umbilical Cord? NeoReviews. 2004;5(4):e142-54.

3. Van Rheenen P, Gruschke S, Brabin BJ. Delayed umbilical cord clamping for reducing anaemia in LBW infants implications for developing countries. Ann Trop Paediatr. 2006;26(3):157-67.

4. McDonald SJ, Middleton P. Effect of timing of umbilical cord clamping of term infants on maternal and neonatal outcomes. Cochrane Database Syst Rev. 2008;(2):CD004074.

5. Chaparro CM, Neufeld LM, Tena Alavez G, Eguia-Liz CR, et al. Effect of timing of umbilical cord clamping on iron status in Mexican infants: a randomised controlled trial. Lancet. 2006;367(9527):1997-2004.

6. Levy T, Blickstein I. Timing of cord clamping revisited. J Perinat Med. 2006;34(4):293-7.

7. Ceriani Cernadas JM, Carroli G, Pellegrini L, Otaño L, et al. The effect of timing of cord clamping on neonatal venous hematocrit values and clinical outcome at term: a randomized, controlled trial. Pediatrics. 2006;117(4):e77986.

8. Hutton EK, Hassan ES. Late vs Early Clamping of the Umbilical Cord in Full-term Neonates Systematic Review and Meta-analysis of Controlled Trials. JAMA. 2007;297(11):1241-52.

9. Van Rheenen P, De Moor L, Eschbach S, De Grooth H, et al. Delayed cord clamping and haemoglobin levels in infancy: a randomised controlled trial in term babies. Trop Med Int Health. 2007;12(5):603-16.

10. Raju TN. Timing of umbilical cord clamping after birth for optimizing placental transfusion. Curr Opin Pediatr. 2013;25(2):180-7.

11. Andersson O, Hellström-Westas L, Andersson D, Domellöf M. Effect of delayed versus early umbilical cord clamping on neonatal outcomes and iron status at 4 months: a randomised controlled trial. BMJ. 2011;343:d7157.

12. Mercer JS, McGrath MM, Hensman A, Silver H, et al. Immediate and delayed cord clamping in infants born between 24 and 32 weeks: a pilot randomized controlled trial. J Perinatol. 2003;23(6):446-72.

13. Rabe H, Reynolds G, Diaz-Rossello J. Early versus delayed umbilical cord clamping in preterm infants. Cochrane Database Syst Rev. 2004;(4):CD003248.

14. Ceriani Cernadas JM, Durán P. Commentary, Cochrane review: early versus delayed umbilical cord clamping in preterm infants. WHO Reproductive Health Library. 2006. [Consulta: 19 de octubre de 2016]. Disponible en: http:/ / apps.who.int/rhl/pregnancy_childbirth/childbirth/3rd stage/jccom/en.

15. Oh W, Fanaroff AA, Carlo WA, Donovan EF, et al. Effects of delayed cord clamping in very-low-birth-weight infants. J Perinatol. 2011;31(Suppl 1):S68-71.

16. Kaempf JW, Tomlinson MW, Kaempf AJ, Wu Y, et al. Delayed umbilical cord clamping in premature neonates. Obstet Gynecol. 2012;120(2 Pt 1):325-30.

17. Rabe H, Díaz-Rosselló JL, Duley L, Dowswell T. Effect of timing of umbilical cord clamping and other strategies to influence placental transfusion at preterm birth on maternal and infant outcomes. Cochrane Database Syst Rev. 2012;(8):CD003248.

18. Elimian A, Goodman J, Escobedo M, Nightingale L, et al. Immediate compared with delayed cord clamping in the preterm neonate: a randomized controlled trial. Obstet Gynecol. 2014;124(6):1075-9.

19. Jelin AC, Zlatnik MG, Kuppermann M, Gregorich SE, et al. Clamp late and maintain perfusion (CLAMP) policy: delayed cord clamping in preterm infants. J Matern Fetal Neonatal Med. 2016;29(11):1705-9.

20. Chiruvolu A, Tolia VN, Qin H, Stone GL, et al. Effect of delayed cord clamping on very preterm infants. Am JObstet Gynecol. 2015;213(5):676.e1-7.

21. Salae R, Tanprasertkul C, Somprasit C, Bhamarapravatana $\mathrm{K}$, et al.Efficacy of delayed versus immediate cord clamping in late preterm newborns following normal labor: a randomized control trial. J Med Assoc Thai. 2016;99(Suppl 4):S159-65.

22. Liu LY, Feinglass JM, Khan JY, Gerber SE, et al. Evaluation of introduction of a delayed cord clamping protocol for premature neonates in a high-volume maternity center. Obstet Gynecol. 2017;129(5):835-43.

23. Dicky O, Ehlinger V, Guyard-Boileau B, Assouline C, et al. Clampage tardif du cordon ombilical chez les enfants prématurés nés avant 37 semaines d'aménorrhée : étude observationnelle prospective. Arch Pediatr. 2017;24(2):11825.

24. Committee Opinion No. 684 Summary: Delayed Umbilical Cord Clamping After Birth. Obstet Gynecol. 2017;129(1):2323.

25. BoereI, Roest AA, WallaceE, Ten Harkel AD, et al.Umbilical blood flow patterns directly after birth before delayed cord clamping. Arch Dis Child Fetal Neonatal Ed. 2015;100(2):F1215.

26. Mercer JS, Erickson-Owens DA, Vohr BR, Tucker RJ, et al. Effects of placental transfusion on neonatal and 18 month outcomes in preterm infants: a randomized controlled trial. J Pediatr. 2016;168:50-5.e1.

27. Nevill E, Meyer MP. Effect of delayed cord clamping (DCC) on breathing and transition at birth in very preterm infants. Early Hum Dev. 2015;91(7):407-11.

28. Tarnow-Mordi W, Morris J, Kirby A, Robledo K, et al. Australian Placental Transfusion Study Collaborative Delayed versus immediate cord clamping in preterm infants. N Engl J Med. 2017;377(25):2445-55.

29. Chiruvolu A, Qin H, Nguyen ET, Inzer RW. The effect of delayed cord clamping on moderate and early late-preterm infants. Am J Perinatol. 2018;35(3):286-91.

30. Fenton C, McNinch NL, Bieda A, Dowling D, et al. Clinical outcomes in preterm infants following institution of a delayed umbilical cord clamping practice change. Adv Neonatal Care. 2018;18(3):223-31.

31. Garabedian C, Rakza T, Drumez E, Poleszczuk M, et al. Benefits of delayed cord clamping in red blood cell alloimmunization. Pediatrics. 2016;137(3):e20153236.

32. Fogarty M, Osborn DA, Askie L, Seidler AL, et al. Delayed vs early umbilical cord clamping for preterm infants: a 
systematic review and meta-analysis. Am J Obstet Gynecol. 2018;218(1):1-18.

33. Duley L, Dorling J, Pushpa-Rajah A, Oddie S, et al. Randomised trial of cord clamping and initial stabilisation at very preterm birth. Arch Dis Child Fetal Neonatal Ed. 2018;103(1):F6-14.

34. Qian Y, Ying X, Wang P, Lu Z, et al. Early versus delayed umbilical cord clamping on maternal and neonatal outcomes. Arch Gynecol Obstet. 2019;300(3):531-43.

35. Katheria AC, Rich WD, Bava S, Lakshminrusimha S. Placental Transfusion for Asphyxiated Infants. Front Pediatr. 2019;7:473.

36. Das B, Sundaram V, Kumar P, Mordi WT, et al. Effect of placental transfusion on iron stores in moderately preterm neonates of 30-33 weeks gestation. Indian J Pediatr. 2018;85(3):172-8.

37. Chopra A, Thakur A, Garg P, Kler N, et al. Early versus delayed cord clamping in small for gestational age infants and iron stores at 3 months of age-a randomized controlled trial. BMC Pediatr. 2018;18(1):234.

38. Andersson O, Hellstrom-Westas L, Domellof M. Elective caesarean: does delay in cord clamping for $30 \mathrm{~s}$ ensure sufficient iron stores at 4 months of age? A historical cohort control study. BMJ Open. 2016;6(11):e012995.

39. Gupta R, Ramji S. Effect of delayed cord clamping on iron stores in babies born to anemic mothers: a randomized controlled trial. Indian Pediatr. 2002;39(2):130-5.

40. Kc A, Rana N, Målqvist M, Jarawka Ranneberg L, et al. Effects of Delayed Umbilical Cord Clamping vs Early Clamping on Anemia in Infants at 8 and 12 Months: A Randomized Clinical Trial. JAMA Pediatr. 2017;171(3):26470.
41. Katheria AC, Truong G, Cousins L, Oshiro B, etal. Umbilical cord milking versus delayed cord clamping in preterm infants. Pediatrics. 2015;136(1):61-9.

42. Shirk SK, Manolis SA, Lambers DS, Smith KL. Delayed clamping vs milking of umbilical cord in preterm infants: a randomized controlled. Am JObstet Gynecol. 2019;220(5):482. e1-8.

43. Katheria A, Reister F, Essers J, Mendler M, et al. Association of Umbilical Cord Milking vs Delayed Umbilical Cord Clamping With Death or Severe Intraventricular Hemorrhage Among Preterm Infants. JAMA. 2019;322(19):1877-86.

44. Balasubramanian H, Ananthan A, Jain V, Rao S, et al. Umbilical cord milking in preterm infants: a systematic review and meta-analysis. Arch Dis Child Fetal Neonatal Ed. 2020;105(6):572-80.

45. Kluckow M, Hooper SB. Using physiology to guide time to cord clamping. Semin Fetal Neonatal Med. 2015;20(4):22531.

46. Ceriani Cernadas JM. La transferencia de células madres al recién nacido mediante la trasfusión placentaria a través del clampeo demorado del cordón umbilical. Arch Argent Pediatr. 2016;114(6):498-9.

47. Lawton C, Acosta S, Watson N, Gonzales-Portillo C, el al. Enhancing endogenous stem cells in the newborn via delayed umbilical cord clamping. Neural Regen Res. 2015;10(9):1359-62.

48. Tolosa JN,ParkDH, Eve DJ, KlaskoSK, et al. Mankind's first natural stem cell transplant. J Cell Mol Med. 2010;14(3):48895. 